\title{
Chimera states induced by spatially modulated delayed feedback
}

\author{
Oleh E. Omel'chenko, ${ }^{1,2,3}$ Yuri L. Maistrenko, ${ }^{3}$ and Peter A. Tass ${ }^{2,4,5}$ \\ ${ }^{1}$ Weierstraß Institute for Applied Analysis and Stochastics, Mohrenstr. 39, 10117 Berlin, Germany \\ ${ }^{2}$ Institute of Neuroscience and Medicine 7-Neuromodulation, Research Center Jülich, 52425 Jülich, Germany \\ ${ }^{3}$ Institute of Mathematics, National Academy of Sciences of Ukraine, Tereshchenkivska Str. 3, 01601 Kyiv, Ukraine \\ ${ }^{4}$ Department of Stereotaxic and Functional Neurosurgery, University of Cologne, 50924 Cologne, Germany \\ ${ }^{5}$ Brain Imaging Center West, 52425 Jülich, Germany
}

(Received 12 September 2010; published 2 December 2010)

\begin{abstract}
Recently, we have presented spatially modulated delayed feedback as a novel mechanism, which generically generates chimera states, remarkable spatiotemporal patterns in which coherence coexists with incoherence [O. E. Omel'chenko et al., Phys. Rev. Lett. 100, 044105 (2008)]. Remarkably, such chimera states serve as a natural link between completely coherent states and completely incoherent states. So far, we have studied this mechanism with a self-consistency-based numerical analysis only. In contrast, in this paper we perform a thorough dynamical description and, in particular, a stability analysis of the emerging chimera states. For this, we apply a recently developed reduction procedure [A. Pikovsky and M. Rosenblum, Phys. Rev. Lett. 101, 264103 (2008)]. By combining analytical and numerical approaches, we systematically describe the relationship between the parameters of the delayed feedback on one hand and the properties of the chimera states on the other hand. We provide the general rules for an effective control and manipulation of the chimera states.

DOI: 10.1103/PhysRevE.82.066201

PACS number(s): 05.45.Xt, 89.75.Kd
\end{abstract}

\section{INTRODUCTION}

Synchronization of coupled systems is a generic mechanism in physics, chemistry, biology, and medicine [1-8]. Numerous studies have been devoted to the transition between synchronous and asynchronous regimes [1-6,9-13]. The vast majority of these studies were devoted to globally or locally coupled systems, as opposed to systems with intermediate nonlocal coupling, which remained out of focus. Finally, Kuramoto and colleagues [14-16] drew the attention to the fact that in many real applications nonlocal coupling is responsible for the emergence of a new peculiar phenomenon. They showed that a symmetric system of identical but nonlocally coupled oscillators can surprisingly exhibit nonhomogeneous partially synchronized spatiotemporal regimes. Such regimes, later denoted as chimera state by Abrams and Strogatz $[17,18]$, are characterized by the coexistence of spatial domains with synchronized oscillators directly neighboring spatial domains with desynchronized oscillators [14-26]. At that time nothing like that had ever been observed in studies on pattern formation and synchronization. Although the notion of chimera states was initially introduced with respect to spatially homogeneous systems only, later similar spatiotemporal patterns were found in different heterogeneous systems as well $[22,27-32]$.

Note that chimeras induced by nonlocal coupling coexist with linearly stable coherent states, and one has to prepare quite specific initial conditions to approach them [14-25]. In contrast, recently we have shown that chimeras generically emerge in networks of globally coupled oscillators starting from a trivial synchronized initial condition, provided the oscillators are exposed to delayed feedback stimulation with a spatially decaying stimulation profile [27]. Delayed feedback stimulation is a standard means for the control of synchronization processes [33-40]. A spatially decaying stimulation profile is in a way generic when local control forces act on spatially extended systems (see, e.g., [41]). Moreover, we have found that the generically emerging chimeras caused by spatially decaying delayed feedback stimulation serve as a natural link between the coherent and the incoherent states [27]. In addition, contrary to nonlocal coupling the delayed feedback stimulation causes a pronounced multistability $[42,43]$. However, to a large extent our previous chimera analysis was based on numerical simulations [27]. To put these findings on a mathematically solid ground, we perform here a strict dynamical analysis and, in particular, a thorough stability and bifurcation analysis. For this, we use a reduction technique recently developed by Pikovsky and Rosenblum [44] and provide a universal dynamical description of the chimera states and other related solutions.

Since we consider the spatially modulated delayed feedback as a control tool, we describe in detail how the parameters of the delayed feedback impact the properties of the generated chimera states. Our results show, in particular, that by adjusting the amplification and the delay of the feedback loop one can effectively control the size of the coherent vs the incoherent domain as well as the distribution of the effective frequencies in the chimera state.

The paper is organized as follows. In Sec. II the model is outlined. We briefly recall the notion of the chimera state and define some auxiliary quantities which are used below for characterization. In Sec. III we apply the method from [44] and derive a reduced integro-differential equation which describes the long-time dynamical behavior of our model in the thermodynamic limit. We demonstrate that the chimera states as well as the phase-locked solutions correspond to standingwave solutions, while the completely incoherent state corresponds to fixed-point solution. A linear stability analysis of all solutions is performed. In Sec. IV we discuss a general structure of the solution of the equation derived in Sec. III and the corresponding typical bifurcation diagrams. As follows from our study, chimera states can be generated by 
spatially modulated delayed feedback also in the absence of global coupling. This situation is explained in Sec. IV B.

\section{MODEL}

Our basic model is a large ensemble of identical LandauStuart oscillators, representing a normal form of a supercritical Andronov-Hopf bifurcation

$$
\begin{aligned}
\dot{W}_{k}(t)= & {\left[r^{2}+i \omega-\left|W_{k}(t)\right|^{2}\right] W_{k}(t)+C\left[\bar{W}(t)-W_{k}(t)\right] } \\
& +K \rho\left(x_{k}\right) \bar{W}(t-\tau), \quad k=1, \ldots, N .
\end{aligned}
$$

Here, $W_{k}(t)$ denotes the position of the $k$ th oscillator in the complex plane, while positive constants $r$ and $\omega$ stand for the radius of the unperturbed limit cycle and the natural frequency of the oscillators, respectively. The oscillators are assumed to be globally coupled (with coupling strength $C \geq 0$ ) and exposed to spatially modulated delayed feedback (with delay $\tau$ and strength of the delayed feedback $K \geq 0$ ). These two types of interaction between oscillators are expressed in terms of the ensemble's mean field

$$
\bar{W}(t):=\frac{1}{N} \sum_{j=1}^{N} W_{j}(t) .
$$

Further, we assume that all oscillators are uniformly distributed over the interval $[-1,1]$, and hence each of them has a prescribed space position

$$
x_{k}=-1+2(k-1) /(N-1), \quad k=1, \ldots, N .
$$

In such settings, the term spatial modulation means that the delayed feedback signal in Eq. (1) is delivered to each oscillator with the spatial profile factor $\rho(x)$, which is supposed to be a non-negative even function, strictly decreasing on $[0,1]$ and obeying the normalization condition

$$
\frac{1}{2} \int_{-1}^{1} \rho(x) d x=1 \text {. }
$$

As a default stimulation profile in what follows we use the exponential stimulation profile defined by

$$
\rho(x)=\frac{a \exp (-a|x|)}{1-\exp (-a)}, \quad \text { with } a>0 .
$$

In many real physical systems the position of an oscillator on its limit cycle provides the relevant information rather than the shape of the oscillator's limit cycle. Accordingly, the phase reduction method [1] is the standard procedure to study the dynamics of oscillator networks, especially their synchronization behavior. In our case, the phase reduction can rigorously be performed if, for example, coupling and delayed feedback are both weak. Then the oscillators' amplitudes can be eliminated $\left[\left|W_{k}(t)\right| \rightarrow r\right]$, and the complex Eq. (1) is reduced $[1,45]$ to the scalar equation of the phase dynamics,

$$
\begin{aligned}
\dot{\Psi}_{k}(t)= & \omega-\frac{C}{N} \sum_{j=1}^{N} \sin \left[\Psi_{k}(t)-\Psi_{j}(t)\right] \\
& -\frac{K}{N} \sum_{j=1}^{N} \rho\left(x_{k}\right) \sin \left[\Psi_{k}(t)-\Psi_{j}(t-\tau)\right], \quad k=1, \ldots, N,
\end{aligned}
$$

where $\Psi_{k}(t)$ is the phase of the $k$ th oscillator.

The analog of Eq. (5) with spatially uniform stimulation profile $[\rho(x) \equiv 1]$ has been analyzed for both vanishing coupling $(C=0)[33]$ and positive coupling $(C>0)$ [34]. Interestingly, both studies revealed either completely coherent or completely incoherent stable states. Later, spatially nonhomogeneous phase-locked solutions induced by a spatially varying stimulation profile were described by $[39,40]$.

In contrast to these studies, in numerical simulations of Eq. (5) with spatially decaying stimulation profile $\rho(x)$ we observed chimera states (see Fig. 1). From a dynamical standpoint the latter are completely different states in that the ensemble of identical oscillators is split into two spatially localized domains. In the coherent domain around the tip of the stimulation profile the oscillators are phase locked. In the other incoherent domain the oscillators are desynchronized. The incoherent domain comprises those parts of the interval $[-1,1]$, where the stimulation strength is subcritical. Such a behavior can be illustrated by plotting the effective frequencies of the oscillators, i.e.,

$$
\left\langle\dot{\Psi}_{k}(t)\right\rangle=\lim _{t \rightarrow \infty} \frac{\Psi_{k}(t)}{t} .
$$

It turns out that with increasing number of oscillators $N$, the points $\left(x_{k},\left\langle\dot{\Psi}_{k}(t)\right\rangle\right)$ densely fill a well-defined continuous curve, which we call the effective frequency curve and denote it by $\omega_{\text {eff }}(x)$. One can easily recognize two different parts of this curve: the coherent part, where the effective frequencies are identical, and the incoherent part, where the effective frequencies vary. Note that the graph of $\omega_{\text {eff }}(x)$ reflects the averaged velocities of the oscillators, while their actual timedependent dynamics $\dot{\Psi}_{k}(t)$ may be much more complicated (see Fig. 2).

Numerical simulations show that the typical effective frequency curve lies either completely below [Fig. 1(a)] or completely above [Fig. 1(b)] the natural frequency $\omega$. That is why we refer to these two opposite situations as slow and fast chimera states, respectively.

To quantify the effective frequency curve $\omega_{\text {eff }}(x)$ with a finite number of scalars we introduce three parameters:

(1) Synchronization parameter $S$, defined as the relative measure of the coherent part of the curve $\omega_{\text {eff }}(x)$.

(2) Mean effective frequency

$$
\omega_{a}:=\frac{1}{2} \int_{-1}^{1} \omega_{\mathrm{eff}}(x) d x .
$$

(3) Dispersion of the effective frequencies 

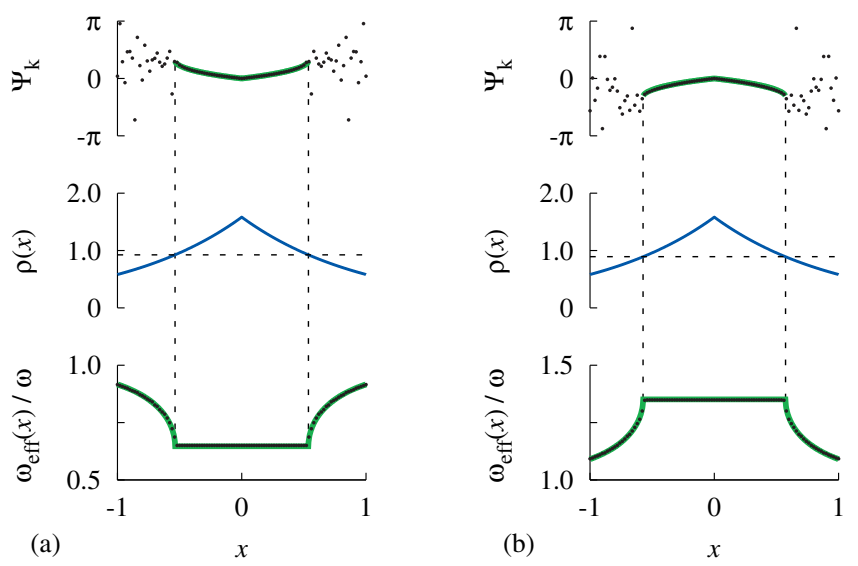

FIG. 1. (Color) Chimera states induced by the delayed feedback with the exponential stimulation profile (4). Parameters: $N=101$, $a=1.0, C=0.1 \pi, K=\pi, \omega=2 \pi$, and (a) $\tau=0.3$ or (b) $\tau=0.6$. System (5) was integrated using a Runge-Kutta method starting from the coherent initial condition. (Top) Snapshot of the oscillators' phases (black dots). (Middle) Stimulation profile. (Bottom) Effective frequencies of the oscillators (black dots). Green thick lines in top and bottom graphs represent the theoretical predictions obtained from Eqs. (20) and (21).

$$
D_{\omega}:=\sqrt{\frac{1}{2} \int_{-1}^{1}\left[\omega_{\mathrm{eff}}(x)-\omega_{a}\right]^{2} d x}
$$

Note that the synchronization parameter $S$ varies between 0 and 1 , and equals 1 for completely phase-locked solutions only.

\section{THERMODYNAMIC LIMIT: ANALYTICAL APPROACH}

One of the ways to treat chimera states analytically was proposed by Kuramoto and Battogtokh [14]. Their approach is based on the self-consistency argument involving a spacedependent mean-field concept. We used this approach in our previous paper [27], where we derived a series of relations connecting the time-averaged parameters of the chimera states (see Eqs. (6)-(8) in [27]). However, this approach is limited since it does not provide a stability analysis. In contrast, in the present paper we utilize another approach developed by Pikovsky and Rosenblum [44]. It reveals the same relations as mentioned above and, hence, confirms our pre-

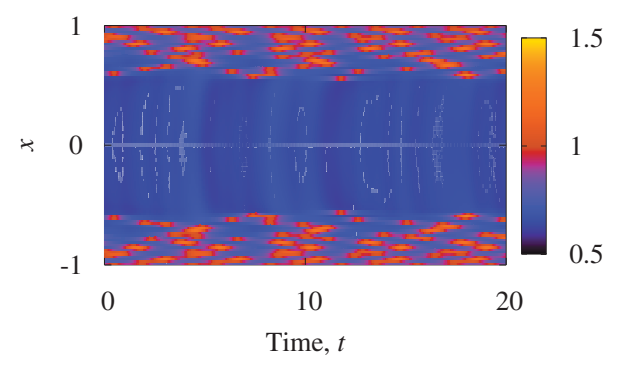

FIG. 2. (Color) Color coded phase velocities along a chimera trajectory corresponding to Fig. 1(a). The dark region is the coherent region. vious results [27]. More importantly, the present approach goes significantly beyond our previous study [27] since it actually enables us to consider the dynamical properties of the chimera states. In fact, it turns out that all bifurcation diagrams presented in [27] have to be modified significantly.

\section{A. Reduction of the system}

First, we rewrite Eq. (5) in the equivalent form

$$
\dot{\Psi}_{k}(t)=\omega+\operatorname{Im}\left[Z_{k}(t) e^{-i \Psi_{k}(t)}\right]
$$

where

$$
Z_{k}(t)=\frac{C}{N} \sum_{j=1}^{N} e^{i \Psi_{j}(t)}+\frac{K}{N} \sum_{j=1}^{N} \rho\left(x_{k}\right) e^{i \Psi_{j}(t-\tau)}
$$

is the effective force acting on the $k$ th oscillator. In the case of the thermodynamic limit, when $N \rightarrow \infty$ and when the oscillators are uniformly distributed over the interval $[-1,1]$ [see Eq. (2)], the spatial profile $\rho(x)$ remains almost constant for all oscillators positioned in a small vicinity of point $x$. Hence, the collective behavior of those oscillators can be characterized by the local complex mean-field $z(x, t)$ defined according to

$$
z(x, t):=\lim _{\substack{N \rightarrow \infty, N \Delta x=\text { const }}} \frac{1}{\tilde{N}(x, \Delta x)} \sum_{j:\left|x_{j}-x\right|<\Delta x} e^{i \Psi_{j}(t)},
$$

where $\tilde{N}(x, \Delta x):=\#\left\{j:\left|x_{j}-x\right|<\Delta x\right\}$. As usual, such a definition implies that the inequality $0 \leq|z(x, t)| \leq 1$ holds for all $x$ and $t$. Moreover, the condition $|z(x, t)|=1$ means that the oscillators around point $x$ are synchronized in phase, while the condition $|z(x, t)|=0$ reflects the local absence of in-phase synchronization. In particular, concerning the chimera state, the relation $|z(x, t)|=1$ identifies the coherent domain, while the inequality $|z(x, t)|<1$ stands for the incoherent domain.

Comparing system (8) and (9) with Eqs. (2) and (3) from [44], we see that they have a similar hierarchical structure, with the space variable $x$ playing the role of a subpopulation index. Therefore, in the thermodynamic limit we can apply the reduction procedure by Pikovsky and Rosenblum [44] and reduce the dynamics of the original system (8) and (9) to a comparatively simpler set of equations.

Without going into detail, which can be found in [44], we mention that the reduction procedure is mainly based on the application of the Watanabe-Strogatz theory [46,47]. In the thermodynamic limit, for solutions of system (8) and (9) this theory suggests the following ansatz:

$$
e^{i \Psi_{k}(t)}=\frac{z\left(x_{k}, t\right) e^{-i \zeta\left(x_{k}, t\right)}+e^{i \varphi_{k}}}{z^{*}\left(x_{k}, t\right) e^{i \varphi_{k}}+e^{-i \zeta\left(x_{k}, t\right)}},
$$

which contains the local mean field $z(x, t)$ as well as an additional real-valued function $\zeta(x, t)$ and $N$ constants $\varphi_{k}$. In the special case when the constants $\varphi_{k}$ are uniformly distributed over the interval $[-\pi, \pi]$, ansatz (11) being substituted in system (8) and (9) produces a delayed integro-differential equation for the local mean field $z(x, t)$, 


$$
\frac{\partial z}{\partial t}=i \omega z+\frac{1}{2} Z(x, t)-\frac{z^{2}}{2} Z^{*}(x, t),
$$

where

$$
Z(x, t)=\frac{C}{2} \int_{-1}^{1} z(y, t) d y+\frac{K}{2} \int_{-1}^{1} \rho(x) z(y, t-\tau) d y,
$$

and the symbol $*$ denotes the complex conjugate. The equation for $\zeta(x, t)$ in this case reads

$$
\frac{\partial \zeta}{\partial t}=\omega+\operatorname{Im}\left[z^{*}(x, t) Z(x, t)\right]
$$

and turns out to decouple from Eqs. (12) and (13). Therefore, the dynamical properties of the resulting system (12)-(14) are determined by Eqs. (12) and (13) only.

Recent findings of Ott and Antonsen [48,49], later generalized in several directions by Laing $[21,22]$, suggest that the dynamical evolution of a large size system (8) and (9) is, in an appropriate physical sense, time-asymptotically attracted toward a reduced manifold given by system (12)-(14) and ansatz (11) with uniformly distributed constants $\varphi_{k}$. In particular, below we are going to demonstrate that all macroscopic properties of the chimera states as well as the typical bifurcation scenarios involving them can be completely explained in terms of the solutions of Eqs. (12) and (13). Note that Eqs. (12) and (13) constitute a significant simplification, compared with system (8) and (9). Indeed, for any statistically stationary solution of system (8) and (9), which may be very different for neighboring indices $k$ (consider, for example, the snapshot of the chimera state in Fig. 1), the corresponding mean-field solution $z(x, t)$ of Eqs. (12) and (13) is expected to be a continuous function of both $x$ and $t$ due to definition (10).

Note that Eqs. (12) and (13) can also be derived in a different way, which is based on the Ott-Antonsen method $[48,49]$ with the probabilistic interpretation of the function $z(x, t)$. This version of the reduction procedure with a variety of examples, including our system (5), was recently formulated by Laing in [22]. In contrast to our study, Laing chose the natural frequencies of the oscillators from a Lorentzian distribution, rather than having them identical. Then, starting from the continuum limit equation he derived the dissipative equivalent of Eqs. (12) and (13), with a term $(i \omega-D) z$ rather than $i \omega z$, where $D$ is a positive real number. Such a modification results in the fact that Eqs. (12) and (13) now has genuine attractors, rather than the neutrally stable solutions described below. It is straightforward to modify our stability calculations (Secs. III C and III D) to show that this is the case.

\section{B. Standing-wave solutions}

We seek for solutions of system (12) and (13) that take the form of standing waves,

$$
z(x, t)=\bar{z}(x) e^{i \Omega t} .
$$

Substituting this ansatz into relation (13), we obtain

$$
Z(x, t)=\left[C+K \rho(x) e^{-i \Omega \tau}\right] R e^{i \Omega t},
$$

where due to the rotational invariance of system (12) and (13) we have assumed that the value of the integral

$$
R=\frac{1}{2} \int_{-1}^{1} \bar{z}(x) d x
$$

is a positive real number [then, obviously, the inequality $0 \leq R \leq 1$ holds since $0 \leq|z(x, t)| \leq 1]$.

Substituting expressions (15) and (16) into Eq. (12) and canceling then the factor $e^{i \Omega t}$ from both sides, we obtain the quadratic equation

$$
\begin{aligned}
{[C} & \left.+K \rho(x) e^{i \Omega \tau}\right] R \bar{z}^{2}(x)-2 i(\omega-\Omega) \bar{z}(x)-\left[C+K \rho(x) e^{-i \Omega \tau}\right] R \\
& =0 .
\end{aligned}
$$

Its solution can be written in the explicit form

$$
\bar{z}(x)=\frac{C+K \rho(x) e^{-i \Omega \tau}}{\left|C+K \rho(x) e^{-i \Omega \tau}\right|}\left[i g(x, R, \Omega)+\sqrt{1-g^{2}(x, R, \Omega)}\right],
$$

where

$$
g(x, R, \Omega)=\frac{\omega-\Omega}{R \mid C+K \rho(x) e^{-i \Omega \tau \mid}} .
$$

Inserting further $\bar{z}(x)$ from Eq. (18) into Eq. (17) we obtain finally the self-consistency equation

$$
R=\frac{1}{2} \int_{-1}^{1} \frac{C+K \rho(x) e^{-i \Omega \tau}}{\left|C+K \rho(x) e^{-i \Omega \tau}\right|}\left[\operatorname{ig}(x, R, \Omega)+\sqrt{1-g^{2}(x, R, \Omega)}\right] d x,
$$

which is identical to Eq. (7) in [27]. Note that values from the same leaf of the complex square-root function should be present in the integrand of Eq. (19), i.e., for any real $y$ we have

$$
\sqrt{1-y^{2}}+i y=H(y)= \begin{cases}\sqrt{1-y^{2}}+i y, & |y| \leq 1 \\ i\left[y-\operatorname{sgn}(y) \sqrt{y^{2}-1}\right], & |y|>1 .\end{cases}
$$

Suppose that we have found a pair of numbers $(R, \Omega)$ solving Eq. (19), then formulas (15) and (18) imply that $|z(x, t)|=1$ if $|g(x, R, \Omega)|<1$, and $|z(x, t)|<1 \quad$ if $|g(x, R, \Omega)|>1$. Hence, equation $|g(x, R, \Omega)|=1$ identifies the boundary between the coherent and incoherent domains of chimera state. In particular, when $|g(x, R, \Omega)|<1$ for all $|x| \leq 1$, then $z(x, t)$ corresponds to a phase-locked solution.

Taking into account ansatz (11), we see that the dynamics of the coherent oscillators $[|z(x, t)|=1]$ is represented by $\arg z(x, t)$. More precisely, the motion of such oscillators is locked, i.e., their phase deviation with respect to the macroscopic phase $\Omega t$ is time independent and given by

$$
\arg \bar{z}(x)=\arg \left[C+K \rho(x) e^{-i \Omega \tau}\right]+\sin ^{-1} g(x, R, \Omega)
$$

(see Eq. (6) in [27]).

Taking into account definition (10) and comparing Eq. (8) with Eq. (14), we see that the dynamics of the variable $\zeta(x, t)$ actually reflects the locally averaged dynamics of the phase 
oscillators positioned around point $x$. Therefore, it is not surprising that by estimating the right-hand side of Eq. (14) we obtain the value of the effective frequency $\omega_{\text {eff }}(x)$. More precisely, substituting ansatz (15) and (16) together with the explicit solution (18) into the right-hand side of Eq. (14), after straightforward transformations we obtain

$$
\begin{aligned}
\omega+ & \operatorname{Im}\left[z^{*}(x, t) Z(x, t)\right] \\
& = \begin{cases}\Omega, & |g(x, R, \Omega)| \leq 1 \\
\Omega+(\omega-\Omega) \sqrt{1-g^{-2}(x, R, \Omega)}, & |g(x, R, \Omega)|>1,\end{cases}
\end{aligned}
$$

which is identical to the expression of $\omega_{\text {eff }}(x)$ given by Eq. (8) in [27].

Summarizing the above results, we conclude that the standing-wave solutions of Eqs. (12) and (13) correspond to the phase-locked and chimera states of the initial Eq. (5). All relations obtained by means of Kuramoto's self-consistency argument are completely reproduced via the analysis of Eqs. (12) and (13).

\section{Linear stability analysis of standing-wave solutions}

We are going to study the stability properties of solutions of Eq. (5), at least on the reduced manifold where Eqs. (12) and (13) are defined. For this, we linearize Eqs. (12) and (13) around the solution $z(x, t)$ and obtain the equation

$$
\frac{\partial u}{\partial t}=\left[i \omega-z(x, t) Z^{*}(x, t)\right] u+\frac{1}{2} U(x, t)-\frac{z^{2}(x, t)}{2} U^{*}(x, t),
$$

where

$$
U(x, t)=\frac{C}{2} \int_{-1}^{1} u(y, t) d y+\frac{K}{2} \int_{-1}^{1} \rho(x) u(y, t-\tau) d y .
$$

Substituting ansatz (15) and expression (16) into Eq. (22), and introducing a dynamical variable

$$
v(x, t)=e^{-i \Omega t} u(x, t),
$$

we rewrite Eqs. (22) and (23) as follows:

$$
\begin{aligned}
\frac{\partial v}{\partial t}= & \mu(x) v+\frac{1}{2}\left[C V(t)+K \rho(x) e^{-i \Omega \tau} V(t-\tau)\right] \\
& -\frac{\bar{z}^{2}(x)}{2}\left[C V^{*}(t)+K \rho(x) e^{i \Omega \tau} V^{*}(t-\tau)\right],
\end{aligned}
$$

where

$$
\begin{gathered}
V(t)=\frac{1}{2} \int_{-1}^{1} v(x, t) d x, \\
\mu(x)=i(\omega-\Omega)-z(x, t) Z^{*}(x, t) \\
=-\sqrt{\left|C+K \rho(x) e^{-i \Omega \tau}\right|^{2} R^{2}-(\omega-\Omega)^{2}}
\end{gathered}
$$

[the latter relation is a consequence of formulas (16) and (18)]. Because of the presence of complex conjugate quanti- ties in Eq. (24), one should consider this complex equation as a system of two coupled linear delay integro-differential equations:

$$
\frac{\partial \mathbf{v}}{\partial t}=\mathbf{M}(x) \mathbf{v}(x, t)+\frac{C}{2} \mathbf{A}(x) \mathbf{V}(t)+\frac{K}{2} \rho(x) \mathbf{B}(x) \mathbf{V}(t-\tau),
$$

where we used the bold symbols for the vector functions

$$
\mathbf{v}=\left(\begin{array}{c}
\operatorname{Re} v \\
\operatorname{Im} v
\end{array}\right), \quad \mathbf{V}=\left(\begin{array}{c}
\operatorname{Re} V \\
\operatorname{Im} V
\end{array}\right)
$$

and for the $(2 \times 2)$ matrices

$$
\begin{gathered}
\mathbf{M}(x)=\left(\begin{array}{cc}
\operatorname{Re} \mu(x) & -\operatorname{Im} \mu(x) \\
\operatorname{Im} \mu(x) & \operatorname{Re} \mu(x)
\end{array}\right), \\
\mathbf{A}(x)=\left(\begin{array}{cc}
1-\operatorname{Re} \bar{z}^{2}(x) & -\operatorname{Im} \bar{z}^{2}(x) \\
-\operatorname{Im} \bar{z}^{2}(x) & 1+\operatorname{Re} \bar{z}^{2}(x)
\end{array}\right), \\
\mathbf{B}(x)=\left(\begin{array}{cc}
\operatorname{Re}\left\{\left[1-\bar{z}^{2}(x)\right] e^{i \Omega \tau}\right\} & \operatorname{Im}\left\{\left[1-\bar{z}^{2}(x)\right] e^{i \Omega \tau}\right\} \\
-\operatorname{Im}\left\{\left[1+\bar{z}^{2}(x)\right] e^{i \Omega \tau}\right\} & \operatorname{Re}\left\{\left[1+\bar{z}^{2}(x)\right] e^{i \Omega \tau}\right\}
\end{array}\right) .
\end{gathered}
$$

According to the theory of delay differential equations with periodic coefficients [50], the stability of solution (18) is determined by its characteristic exponents. To find them, we substitute the ansatz $\mathbf{v}(x, t)=\overline{\mathbf{v}}(x) e^{\lambda t}$ into the linearized Eq. (27) and obtain the eigenvalue problem

$$
(\lambda \mathbf{I d}-\mathbf{M}(x)) \overline{\mathbf{v}}(x)=\left(\frac{C}{2} \mathbf{A}(x)+\frac{K}{2} \rho(x) e^{-\lambda \tau} \mathbf{B}(x)\right) \frac{1}{2} \int_{-1}^{1} \overline{\mathbf{v}}(x) d x .
$$

This problem can be analyzed as follows. First, we discretize Eqs. (12) and (13) replacing the continuous function $z(x, t)$ with a sequence of functions $z\left(x_{k}, t\right)$, with $k=1, \ldots, N$. Accordingly, we replace the integration $\int_{-1}^{1} d x$ with the summation $\frac{2}{N} \sum_{k=1}^{N}$. In this way, we write the discrete version of the self-consistency equation (19) and the corresponding discrete version of the spectral problem (28). The latter has a form of a common $\mathrm{N}$-dimensional eigenvalue problem and can be solved using standard routines provided by MATLAB or other computational software. A typical spectrum revealed by such a procedure consists of two parts: a discrete spectrum $\sigma_{d}$, with elements which have individual limit behavior as $N \rightarrow \infty$, and a pseudocontinuous spectrum $\sigma_{p c}$ with elements which accumulate in the thermodynamic limit on a curve in the complex plane (see Fig. 5). Such a behavior seems to be natural for coupled oscillators models and was previously reported in $[51,52]$.

Note that the location of the accumulation set of the pseudocontinuous spectrum $\sigma_{p c}$ can be explained by a simple argument. Let us consider an idealized local perturbation at one fixed point $x$ only. Then the integral term in Eq. (28) vanishes, and we get the simple matrix equation $[\lambda \mathbf{I d}-\mathbf{M}(x)] \overline{\mathbf{v}}(x)=0$ with $x$ being a parameter. Solving the corresponding characteristic equation 


$$
\operatorname{det}[\lambda \mathbf{I} \mathbf{d}-\mathbf{M}(x)]=0,
$$

with different $x \in[-1,1]$, we obtain a set of values $\lambda$ which turns out to fit perfectly to the accumulation set of the pseudocontinuous spectrum $\sigma_{p c}$ obtained numerically. More precisely, the function $\mu(x)$ defined by Eq. (26) is either real or pure imaginary. Hence, for every $x$ Eq. (29) provides either a double real nonpositive eigenvalue [if $\operatorname{Im} \mu(x)=0]$ or a pair of conjugate imaginary eigenvalues [if $\operatorname{Re} \mu(x)=0$ ].

Of course, the above argument is not strictly correct since the pointwise perturbation is equivalent to the zero perturbation in $L^{2}$. But we presume that it can be put on a rigorous base in a way similar to $[53,54]$.

\section{Completely incoherent state}

A common way to study the stability of completely incoherent state is to write a continuous formulation of Eq. (5) in terms of a probability density and then to consider its linearization around the uniform density distribution $[2,53,55]$. However, as follows from the discussion in [44], the same analysis can be done by means of system (12) and (13). In this settings, the completely incoherent state corresponds to the trivial solution of system (12) and (13), i.e. $z(x, t) \equiv 0$. Due to definition (10), this implies the absence of any local, and hence global, in-phase synchronization.

Linearizing Eq. (12) around zero we obtain the equation

$$
\frac{\partial u}{\partial t}=i \omega u+\frac{C}{4} \int_{-1}^{1} u(y, t) d y+\frac{K}{4} \int_{-1}^{1} \rho(x) u(y, t-\tau) d y,
$$

which is equivalent to the vector system

$$
\frac{\partial \mathbf{u}}{\partial t}=\mathbf{N u}(x, t)+\frac{C}{2} \mathbf{U}(t)+\frac{K}{2} \rho(x) \mathbf{U}(t-\tau),
$$

where

$$
\mathbf{u}=\left(\begin{array}{l}
\operatorname{Re} u \\
\operatorname{Im} u
\end{array}\right), \quad \mathbf{U}=\frac{1}{2} \int_{-1}^{1} \mathbf{u}(x, t) d x, \quad \mathbf{N}=\left(\begin{array}{cc}
0 & -\omega \\
\omega & 0
\end{array}\right) .
$$

Substituting the ansatz $\overline{\mathbf{u}}(x) e^{\lambda t}$ into the latter system, we obtain

$$
(\lambda \mathbf{I d}-\mathbf{N}) \overline{\mathbf{u}}=\frac{C}{2} \overline{\mathbf{U}}+\frac{K}{2} \rho(x) e^{-\lambda \tau} \overline{\mathbf{U}}
$$

with

$$
\overline{\mathbf{U}}=\frac{1}{2} \int_{-1}^{1} \overline{\mathbf{u}}(x) d x \text {. }
$$

Now we proceed as in the previous section. First, by considering idealized pointwise perturbations at different $x \in[-1,1]$, we obtain two eigenvalues $\pm i \omega$ with continuum degeneracy. This implies that the completely incoherent state can be neutrally stable only. Integrating Eq. (32) with respect to $x$ and taking into account the normalization condition (3), we then come to the system of two linear equations:

$$
\left[\left(\lambda-\frac{C}{2}-\frac{K}{2} e^{-\lambda \tau}\right) \mathbf{I d}-\mathbf{N}\right] \overline{\mathbf{U}}=0 .
$$

Its characteristic equation,

$$
\left(\lambda-\frac{C}{2}-\frac{K}{2} e^{-\lambda \tau}\right)^{2}+\omega^{2}=0
$$

determines the rest of spectrum. Obviously, the latter is discrete. Moreover, it is easy to verify that both $\lambda$ and $\lambda^{*}$ are roots of Eq. (33), and that zero never solves Eq. (33). Hence, the completely incoherent state loses its stability typically via a Hopf bifurcation. A straightforward analysis of Eq. (33) shows that such a bifurcation occurs at curves

$$
\tau=\tau_{m}^{ \pm}(K)=\frac{4 \pi m \pm 2 \cos ^{-1}\left(-\frac{C}{K}\right)}{2 \omega \mp \sqrt{K^{2}-C^{2}}},
$$

where $K \geq C$ and $m \in \mathbb{Z}$. The completely incoherent state is neutrally stable if and only if all roots of Eq. (33) lie in the left half-plane (LHP). Taking into account that parameters $C$ and $K$ are both positive, the latter condition defines a sequence of dropletlike domains in the control parameter plane (see Figs. 8 and 10) given by the inequality

$$
\tau_{m}^{+}(K)<\tau<\tau_{m+1}^{-}(K),
$$

where $K \geq C$ and

$$
2\left[\pi-\cos ^{-1}\left(-\frac{C}{K}\right)\right]<\frac{\omega}{2 m+1} .
$$

Since the expression on the left-hand side of (35) is an increasing unbounded function of $K$ tending to $\pi C / 2$ as $K \rightarrow C$, inequality (34) defines a finite number of "droplets" in the $(\tau, K)$ plane with $m=0,1,2, \ldots,\left[\omega / \pi C-\frac{1}{2}\right]$, where $[x]$ denotes the largest integer less or equal to $x$. Moreover, the size of the droplet becomes smaller as the delay $\tau$ increases. Note that similar results are typical for any other system with delayed feedback control (for example, compare our Fig. 8 with Fig. 4 in [34], with Fig. 2 in [56], and with Fig. 2 in [57]).

\section{DISCUSSION}

\section{A. Bifurcation analysis}

In our previous paper [27] we have observed that the phase-locked solutions and the chimera states typically constitute a branch of solution of Eq. (5), which naturally links the completely coherent and the completely incoherent states. This and other results were obtained phenomenologically, lacking a strict stability analysis of the derived solutions. In contrast, in the present paper, by considering the thermodynamic limits (12) and (13) and the spectral problem (28) derived in the previous section, we are able to provide a largely rigorous description of the bifurcation scenarios mediating the transition between different types of synchronized and partially synchronized solutions of Eq. (5). 

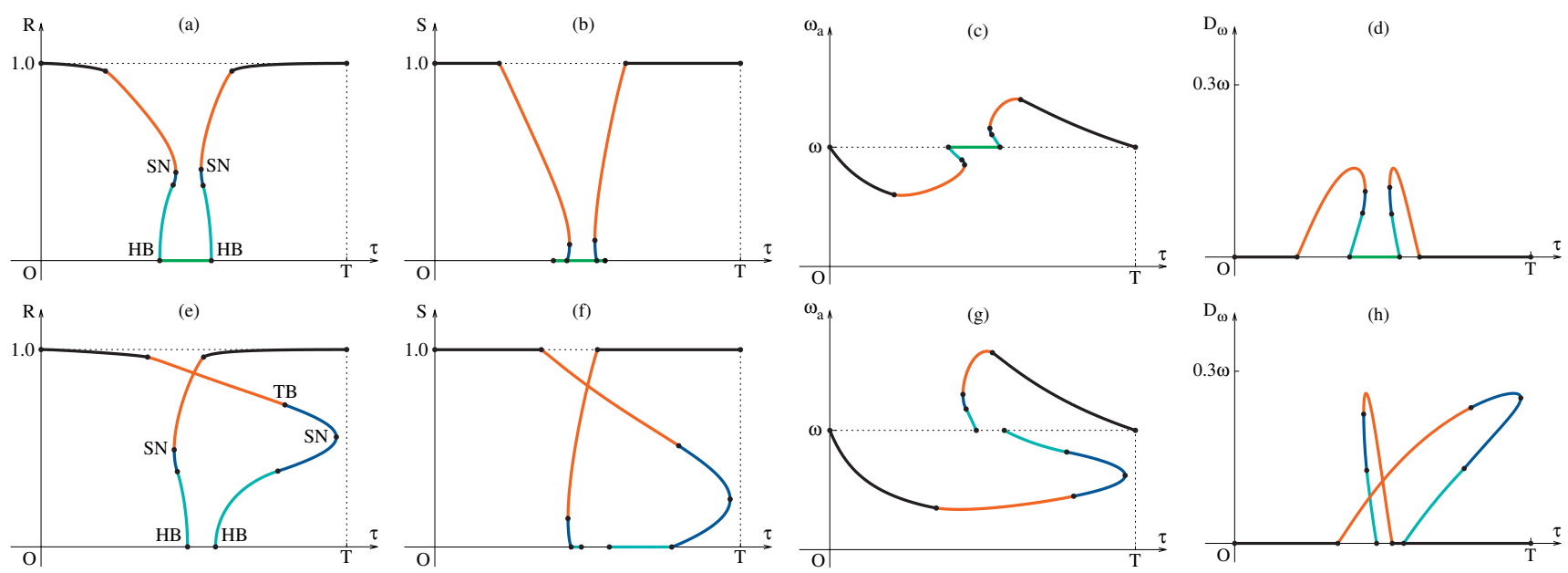

FIG. 3. (Color) Schematic representation of [(a) and (e)] order parameter $R$, [(b) and (f)] synchronization parameter $S$, [(c) and (g)] mean effective frequency of the oscillators $\omega_{a}$, and $\left[(\mathrm{d})\right.$ and (h)] dispersion of the effective frequencies $D_{\omega}$ for two typical solutions of Eq. (19). Parameters: exponential stimulation profile with $a=1.0, C / \omega=0.05$, and (a)-(d) $K / \omega=0.65$ or (e)-(h) $K / \omega=1.1$. Different colors represent stable phase-locked state (black), stable chimera state (red), unstable chimera state (dark blue), unstable drift solution (blue), and stable completely incoherent state (green). The various bifurcation points obtained via the analysis of characteristic equations (29), (28), and (33) are denoted as follows: TB, torus bifurcation; SN, saddle-node bifurcation; HB, Hopf bifurcation. Note that the torus bifurcation is observed for large stimulation strengths only. In contrary, the completely incoherent state is always unstable for large stimulation strengths, while for moderate values of stimulation it can have a stability interval.

The self-consistency equation (19) has one obvious solution $(R=1.0, \Omega=\omega)$, provided $\tau=k T, k \in \mathbb{Z}$, where $T=2 \pi / \omega$ is the natural period of the oscillators. This solution stands for the completely coherent state, when all oscillators rotate synchronously with the same angular frequency $\omega$. Taking this solution as a starting point and varying the time delay $\tau$, we solve Eq. (19) numerically and obtain a continuous curve of the solutions. In parallel, we trace the corresponding spectrum produced by Eq. (28) as it was explained in Sec. III C.

Two typical solutions of Eq. (19) obtained in such a way are shown in Figs. 3(a)-3(d). The corresponding typical profiles of the effective frequencies are plotted in Fig. 4. We start to move along the solution from the point corresponding to the completely coherent state [point with $R=1$ and $\tau=0$ in Fig. 3(a)]. With varying $\tau$, this state, first, survives as a stable phase-locked solution with modified effective frequency and deformed phase pattern [black curve in Figs. 3(a) and 4(a)] (see also [40] for similar results). The stability of such solutions follows directly from their spectral properties [Fig. 5(a)]. Indeed, the corresponding discrete spectrum lies in the LHP [we omit the zero eigenvalue originating from the periodic nature of ansatz (15)], and the pseudocontinuous spectrum accumulates on a bounded interval of the real axis, lying completely on the left of the imaginary axis (compare this with a similar spectral behavior reported in [51]).

For a certain critical value of the delay, the pseudocontinuous spectrum goes to zero, and one enters a region with chimera states [red curve in Figs. 3(a) and 4(b)]. The point dividing black and red curves indicates the boundary between the phase-locked solution and the chimera state. With a further increase in the delay, the pseudocontinuous spectrum of the chimera state gets a T-shaped form [Fig. 5(b)]. According to Eq. (29), the real eigenvalues in the accumula-
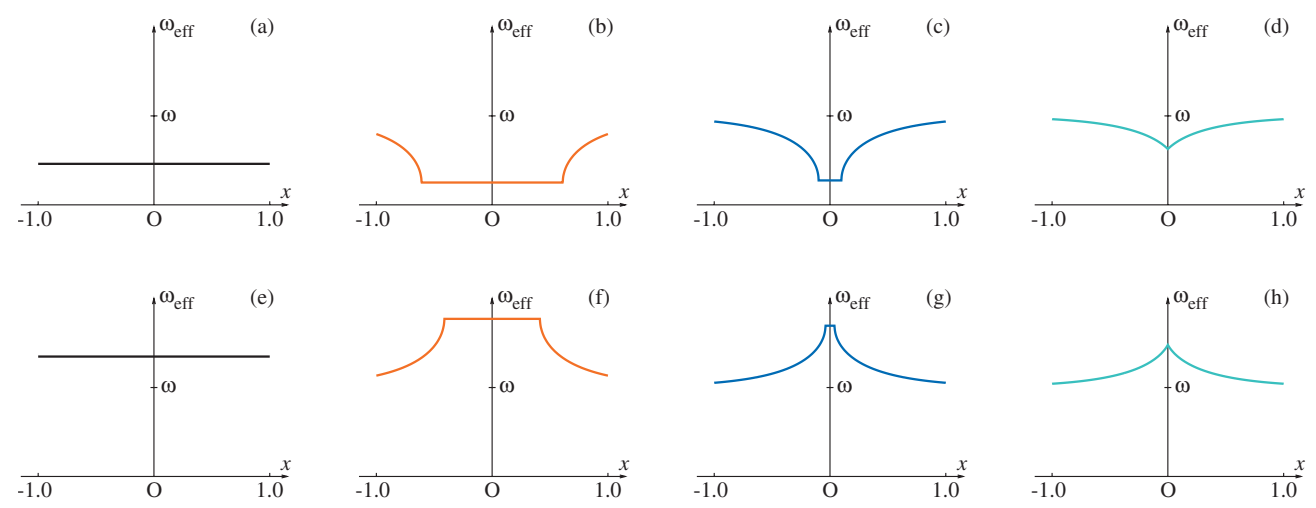

FIG. 4. (Color) Typical effective frequency profiles corresponding to different types of solutions from Fig. 3(a). Moving along the branch of the solution starting from the point $R=1, \tau=0$ in Fig. 3(a), we observe consecutively profiles (a)-(d). Alternatively, if we move along another branch of the solution starting from the point $R=1, \tau=T$, we observe a sequence of profiles (e)-(h). 

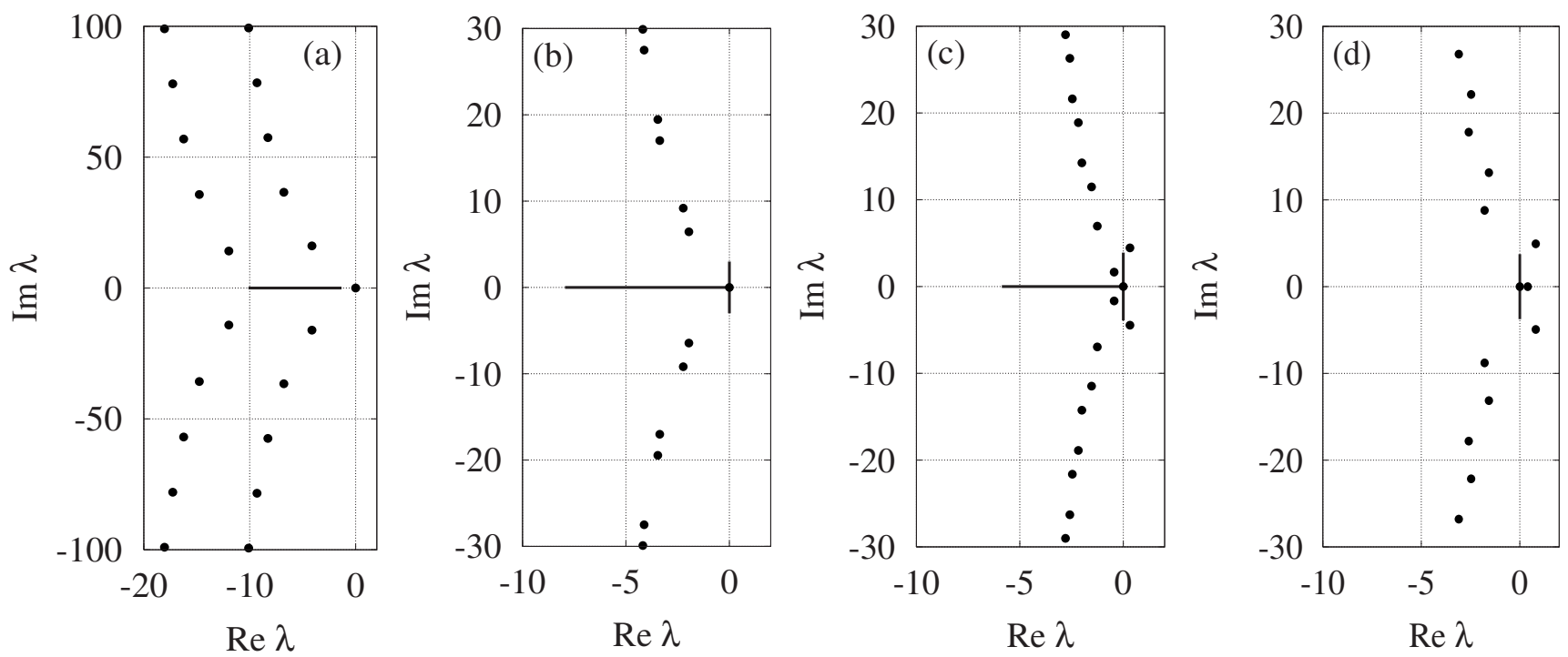

FIG. 5. Typical spectra for the (a) phase-locked state, (b) stable chimera state, (c) unstable chimera state, and (d) unstable completely drifting solution, calculated from Eq. (28) on the solution of Eq. (12). To illustrate the appearance of the torus bifurcation we present here spectra for the branch of the solution which starts from the point $R=1, \tau=0$ in Fig. 3(e). The thick solid lines represent the accumulation set of the pseudocontinuous part of the spectrum given by Eq. (29), while the dots belong to the discrete spectrum given by Eq. (28). Plots (b) and (c) show that the chimera state loses its stability through a torus bifurcation.

tion set correspond to the phase-locked oscillators, while the pairs of conjugate pure imaginary eigenvalues are produced by the drifting ones. Note that the presence of eigenvalues at the imaginary axis usually makes it impossible to perform the linear stability analysis in a rigorous form. Nevertheless, the results of our numerical simulations suggest that the stability of the chimera states mainly depends on the position of the discrete spectrum and is not sensitive to the presence of marginal eigenvalues [58]. In particular, the red curve marks the stable chimera states with discrete spectrum in the LHP [Fig. 5(b)], i.e., those which are observed in numerical simulations. Along this curve the synchronization parameter $S$ varies monotonically with changing delay [see Fig. 3(b)]. This corresponds to a progressive transition of the oscillators from the phase-locked to the drifting state at the edges of the coherence region.

By further increasing the time delay $\tau$ we come to a saddle-node bifurcation point: the stable chimera (red curve) collides with an unstable chimera [dark-blue curve in Figs. 3 (a) and 4(c)]. Further following along the unstable chimera (dark-blue curve) and the unstable completely drifting solution [blue curve in Figs. 3(a) and 4(d)] we finally approach a subcritical Hopf bifurcation point, at which the completely incoherent state $(R=0)$ changes its stability. The latter state has been considered in Sec. III D. From the results presented above [see Eq. (34)] it follows that the completely incoherent state is stable on the interval marked by the green segment in Fig. 3(a). We recall that the completely incoherent state has a stability interval for moderate stimulation strengths $K$ only, while for strong stimulation it is always unstable (see Sec. III D).

In the same way as described above, we construct another branch of the solution originating from the point with $R=1$ and $\tau=T$ in Fig. 3(a). Comparing Figs. 4(a) $-4(\mathrm{~d})$ and Figs. 4(e)-4(h), corresponding to the left and right parts of Fig. 3(a), we see that the effective frequencies curves are either completely retarded (left branch) or completely accelerated (right branch) with respect to their natural frequency $\omega$. In particular, the left branch in Fig. 3(a) contains the slow chimera only, while the right one contains the fast chimera only.

Remarkably, the above dynamical scenario, with the stability of the chimera state being mediated by the saddle-node bifurcation, is observed for moderate values of the stimulation strength $K$ only. If $K$ is large enough [see Fig. 3(e)], then the chimera state loses its stability through a torus bifurcation [59] at the point where a pair of conjugate eigenvalues from the discrete spectrum crosses the imaginary axis [compare Figs. 5(b) and 5(c)]. Note that such a bifurcation cannot be detected via Kuramoto's self-consistency approach. Therefore, we missed it in our previous study [27].

The bifurcation diagrams in Fig. 3 reveal the connection between the chimeras on one hand and the coherent and completely incoherent states on the other hand. To verify this theoretical prediction we compare it with the results of the corresponding numerical simulations. More precisely, we perform a standard dynamical continuation procedure for the solution of system (5) with $N=101$ oscillators. To detect only stable solutions, we supply the right-hand side of each equation of system (5) with an additional Gaussian white noise term $F_{j}(t)$, satisfying $\left\langle F_{j}(t)\right\rangle=0$ and $\left\langle F_{j}(t) F_{k}\left(t^{\prime}\right)\right\rangle$ $=A \delta_{j k} \delta\left(t-t^{\prime}\right)$, where $A$ is a constant noise amplitude (default value is $A=0.0001$ ) [60]. Starting from the completely coherent initial condition, we first increase the time delay $\tau$ in Eq. (5) from zero to $T$, and then decrease it back again. The numerical data obtained in this way perfectly coincide with the theoretical curves shown in Figs. 3(a)-3(h). More importantly, we obtain two hysteresis loops, illustrated schematically in Fig. 6. These loops are bounded by the predicted bifurcation points and indicate the multistability behavior of our system. In particular, we observe that stable chimera 

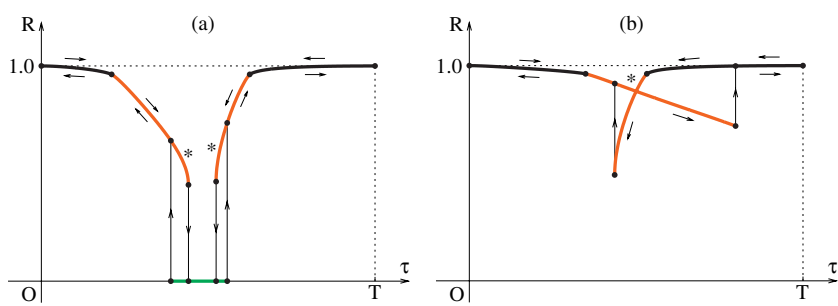

FIG. 6. (Color) Schematic representation of the hysteresis behavior observed in numerical simulations of Eq. (5). Parameters: $a=1.0, C=0.1 \pi, \omega=2 \pi$, and (a) $K=1.3 \pi$ or (b) $K=2.2 \pi$. Different colors on the plot of order parameter $R$ stand for the stable phaselocked state (black line), stable chimera (red line), and stable completely incoherent state (green line). Curves (a) and (b) consists of the stable parts of the curves in Figs. 3(a) and 3(b), respectively. "*” in (a) indicates coexistence of a stable chimera state and a stable completely incoherent state. "*" in (b) indicates coexistence of two different stable chimera states.

state can coexist with the completely incoherent state [“*” in Fig. 6(a)] or two chimera states can exists simultaneously [“*”" in Fig. 6(b)]. Note that since we do not observe any stable solution extending the chimera state beyond the torus bifurcation point in Fig. 6(b), we conjecture that this bifurcation is a subcritical one.

Taking into account the scenario from Fig. 3 and using the same analytical-numerical technique as described above, we can construct the complete solution of Eq. (19) consisting of an infinite sequence of overlapping branches (Fig. 7). In this way, we reveal the main features of all stable chimeras given by Eq. (19) (Fig. 8). As we have mentioned above, the stability region of the chimera state is typically bounded by a curve consisting of two different parts: the saddle-node bifurcation line (for moderate values of $K$ ) and the torus bifurcation line (for large values of $K$ ). We missed the latter in our previous study [27]; therefore, we have indicated a much larger parameter region for stable chimera states than it actually is (compare Figs. 3a and $3 b$ in [27] with their cor-
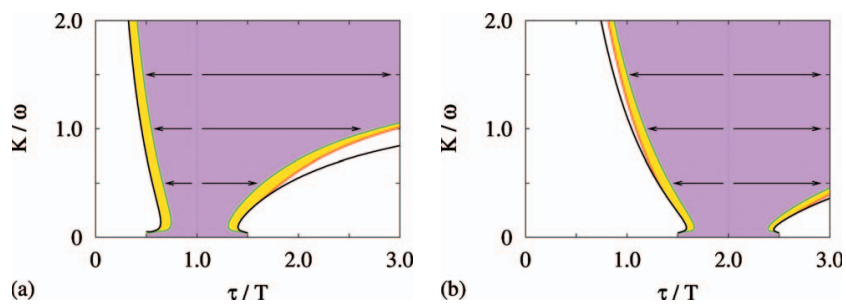

FIG. 7. (Color) Multiple-leaf structure of the solution of Eq. (19) for default parameters: $C / \omega=0.05$ and exponential stimulation profile with $a=1.0$. Starting from the completely synchronized state $(R=1, \Omega=\omega)$ that solves Eq. (19) for $\tau=0, T, 2 T, \ldots$, and continuing the solution into the directions marked with arrows [see (a) and (b)], we obtain different branches of the solution. In the violet region, the solution is a stable phase-locked state, whereas in the yellow region, it is a stable chimera state. At the right (left) edge of each leaf there is a slow (fast) chimera state. Green lines mark the boundary between the phase-locked solutions and the chimera states. Near this line the chimera state is a stable solution and loses its stability via a saddle-node bifurcation (black line) or via a torus bifurcation (red line).
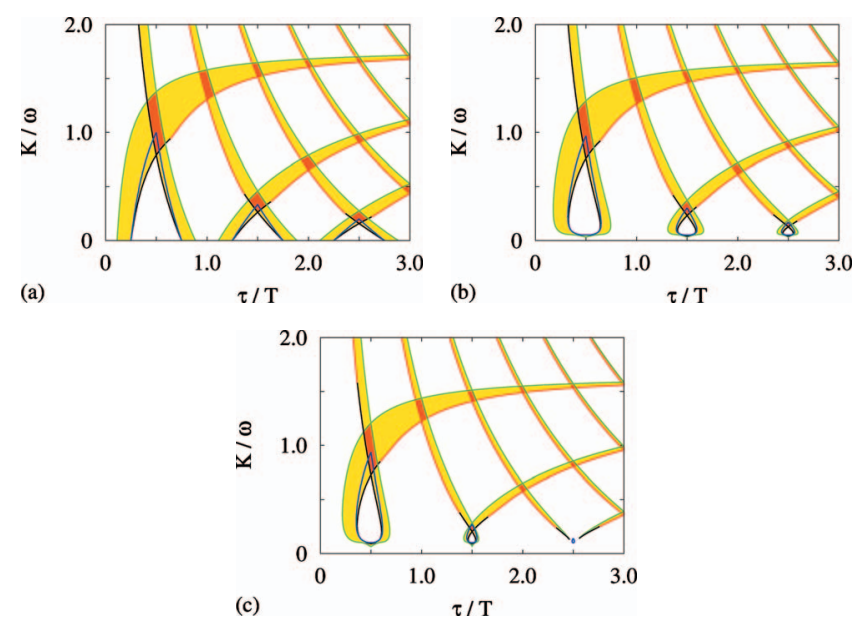

FIG. 8. (Color) Domains in the control parameters plane with one (yellow) or two (orange) stable chimera states. The incoherent state is stable inside the regions marked by blue lines. Other colored lines denote different stability boundaries as explained in the caption of Fig. 7. Note that stable chimera states are not observed for $K=0$ in (a). But they are induced by any arbitrarily small positive stimulation strength $K$, provided the delay $\tau$ lies in the corresponding yellow domain. Parameters: exponential stimulation profile with $a=1.0$, and (a) $C / \omega=0$, (b) $C / \omega=0.05$, or (c) $C / \omega=0.1$.

rected versions Figs. 8(b) and 8(a), respectively).

The parameter dependence of the characteristics of the slow and fast chimera states, order parameter $R$ [Eq. (17)], synchronization parameter $S$ (i.e., the relative measure of the coherent domain), mean effective frequency of the oscillators $\omega_{a}$ [Eq. (6)], and dispersion of the effective frequencies $D_{\omega}$ [Eq. (7)] belonging to Fig. 8(b) are shown in Fig. 9. These plots demonstrate that by a proper adjustment of the feedback parameters one can select particular chimera states out of a wide range of possible dynamical features. In particular, Fig. 8 shows domains in the control parameter plane where one or two stable chimera states (co)exist. Remarkably, the multiple-leaf structure of the constructed solution is very similar to that described in other models with delayed coupling $[56,57]$. Such a reappearance of branches was shown to be a typical feature of any system with delay $[42,43]$. The latter is also responsible for the pronounced multistability observed in our system, where different values of the control parameters $\tau / T$ and $K / \omega$ may be connected with different combinations of coexisting stable solutions (Fig. 10).

The results presented above are sensitive to the value of the coupling strength $C$ (see Fig. 8). In particular, comparing Figs. 8(a)-8(c) we see that chimera states emerge only for $K \geq C$, while for stimulation strengths smaller than the global coupling (i.e., for $K<C$ ) the solutions are phase locked and synchronized. Moreover, the increasing coupling gradually destroys the dropletlike domains at the bottom and shifts the striplike structure to higher values of $K$. Note that the appearance of the torus bifurcation for large stimulation strengths makes spatially modulated delayed feedback less effective, since in this case the properties of the stable chimera states differ slightly from the phase-locked solutions. In particular, one cannot significantly desynchronize the system to obtain small values of the synchronization parameter $S$ or the order parameter $R$. 

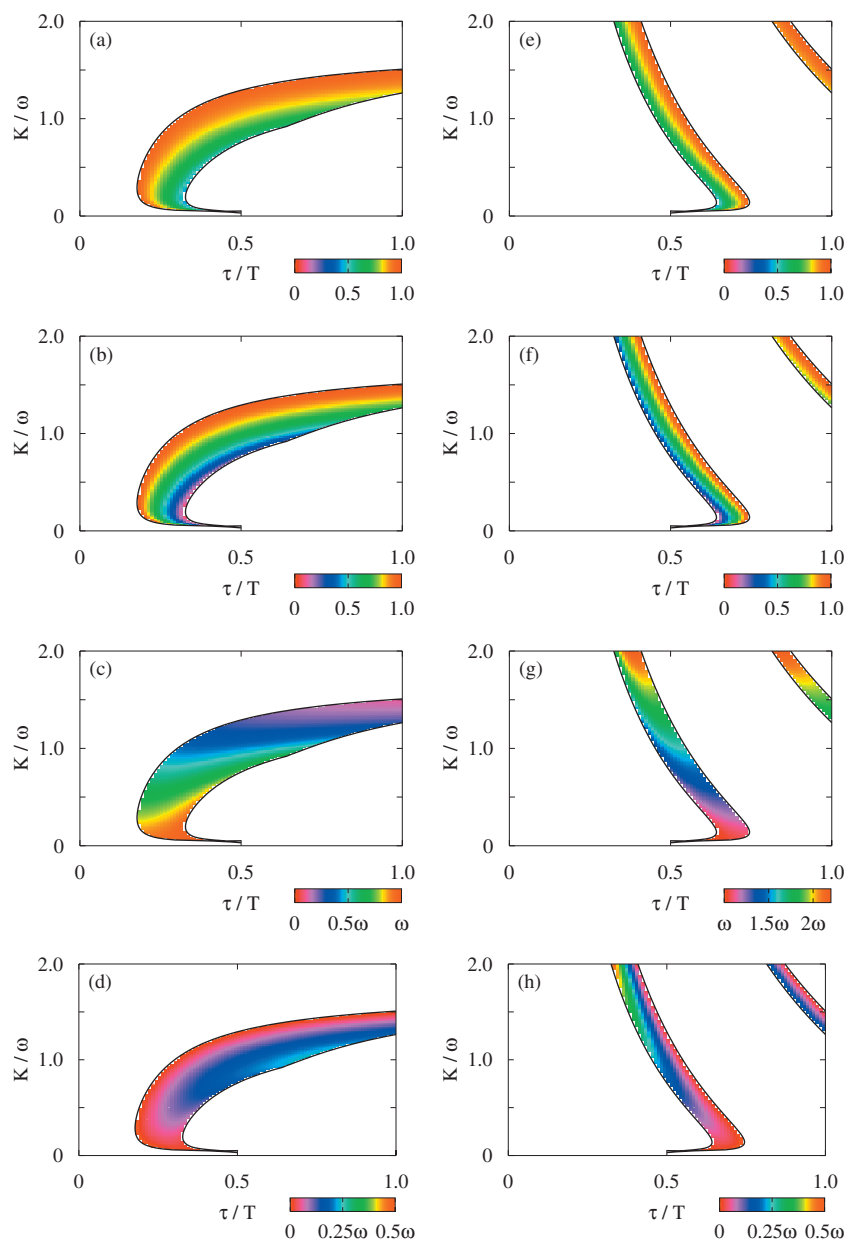

FIG. 9. (Color) Characteristics of (a)-(d) the stable slow chimera and (e)-(h) the stable fast chimera corresponding to the enlargement of the left third of Fig. 8(b): [(a) and (e)] order parameter $R$, [(b) and (f)] synchronization parameter $S,[(\mathrm{c})$ and $(\mathrm{g})]$ mean effective frequency of the oscillators $\omega_{a}$, and $[(\mathrm{d})$ and (h)] dispersion of the effective frequencies $D_{\omega}$.

To study to which extent our results depend on the particular shape of the stimulation profile, we have compared Fig. 8(b) with the corresponding results for the linear stimulation profile

$$
\rho(x)=-a|x|+\left(1+\frac{a}{2}\right), \quad \text { with } 0<a \leq 2,
$$

and for the inverse stimulation profile

$$
\rho(x)=\frac{a}{\tan ^{-1}(1 / a)} \frac{1}{a^{2}+x^{2}}, \quad \text { with } a>0 .
$$

We observe (see Fig. 11) that the bifurcation scenario presented above is robust with respect to variations of the shape of the decaying stimulation profile. However, the linear spatial decay results in the largest area of stable chimera states. Hence, the linear spatial decay seems to be the most suitable choice for a control setup.

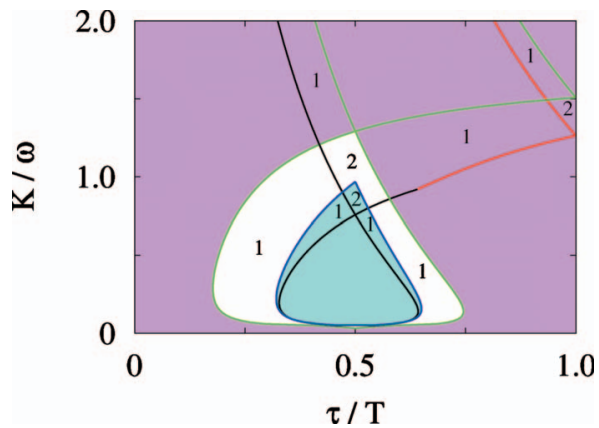

FIG. 10. (Color) An enlargement of the left third of Fig. 8(b) illustrates the multistability of the system under study. See Fig. 8 for an explanation of the colored lines. In the blue region the completely incoherent state is stable. In the violet region, at least one stable coherent solution exists. Numbers indicate the number of stable chimera states in the corresponding subdomains.

\section{B. Delayed stimulation without coupling}

Remarkably, the bifurcation diagrams from Fig. 3 remain qualitatively the same even in the absence of global coupling, i.e., when $C=0$. This underlines that for the emergence of chimera states only the spatially modulated delayed feedback is crucial. The noncoupling case is also interesting from the theoretical point of view, since in this case Eq. (19) becomes significantly simpler and provides more explicit information, which can be used, for example, for an asymptotic analysis of Eq. (5). Indeed, if $C=0$, then Eq. (19) reads

$$
\frac{1}{2} \int_{-1}^{1} H(g(x, R, \Omega)) d x=R e^{i \Omega \tau},
$$

where the definition of function $g$ is modified as follows:

$$
g(x, R, \Omega)=\frac{\omega-\Omega}{R K \rho(x)} .
$$

Since the stimulation profile $\rho(x)$ is assumed to be positive and strictly decreasing on $[0,1]$, the equation

$$
\left|g\left(x_{0}, R, \Omega\right)\right|=1
$$

has at most one solution $x_{0}$ belonging to this interval. Provided this solution exists, it defines the boundary between coherent and incoherent domains of the chimera state. More precisely, the coherent and incoherent domains are given by
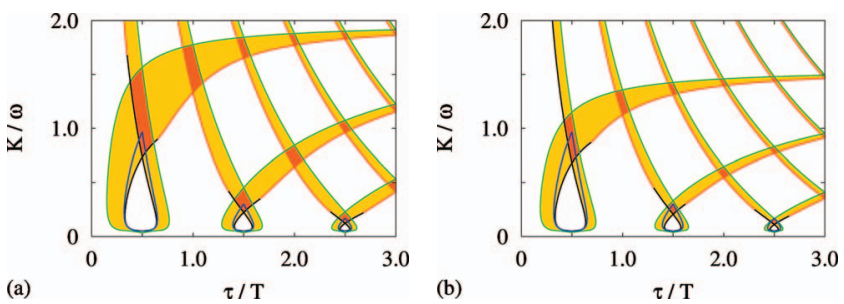

FIG. 11. (Color) Domains in the control parameter plane with one (yellow) or two (orange) stable chimera states. See Fig. 8 for an explanation of the colored lines. Parameters: (a) linear [see Eq. (36)] or (b) inverse [see Eq. (37)] stimulation profile with $a=1.0$ and $C / \omega=0.05$. 
the inequalities $|x|<x_{0}$ and $x_{0}<|x| \leq 1$, respectively.

Let us consider Eqs. (38) and (40) as a system. Taking into account definition (39), we represent this system in the equivalent form

$$
\pm \rho\left(x_{0}\right) \int_{0}^{1} H\left( \pm \frac{\rho\left(x_{0}\right)}{\rho(x)}\right) d x=\frac{\omega-\Omega}{K} e^{i \Omega \tau}, \quad \frac{\omega-\Omega}{R K \rho\left(x_{0}\right)}= \pm 1 .
$$

Introducing the functions

$$
\begin{gathered}
P\left(x_{0}\right)= \pm\left|\rho\left(x_{0}\right) \int_{0}^{1} H\left( \pm \frac{\rho\left(x_{0}\right)}{\rho(x)}\right) d x\right|, \\
Q\left(x_{0}\right)=\arg \left[\rho\left(x_{0}\right) \int_{0}^{1} H\left( \pm \frac{\rho\left(x_{0}\right)}{\rho(x)}\right) d x\right] \in[0,2 \pi),
\end{gathered}
$$

defined for all $x_{0} \in[0,1]$, we obtain

$$
\frac{\omega-\Omega}{K}=P\left(x_{0}\right), \quad \Omega \tau=Q\left(x_{0}\right) .
$$

Now excluding $\Omega$ from system (41), we finally have just one scalar equation

$$
\left[\omega-K P\left(x_{0}\right)\right] \tau=Q\left(x_{0}\right) .
$$

It is easy to see that every solution $x_{0} \in(0,1)$ of Eq. (42) stands for a chimera state. The corresponding values of frequency $\Omega$ and order parameter $R$ can be found in Eqs. (41) and (40), respectively.

One can use these formulas to determine the characteristic boundaries in the control parameter plane. For example, the results of numerical simulations (see Figs. 3 and 4) imply that the boundaries between phase-locked and chimera states are given by

$$
g(1, R, \Omega)= \pm 1
$$

Substituting this condition (i.e., $x_{0}=1$ ) in Eq. (42) we obtain such boundaries in the explicit form

$$
\tau=\frac{q^{ \pm}+2 \pi m}{\omega \mp R^{ \pm} K \rho(1)}, \quad m=0,1,2, \ldots,
$$

where

$$
R^{ \pm}=\left|\int_{0}^{1} H\left( \pm \frac{\rho(1)}{\rho(x)}\right) d x\right|, \quad q^{ \pm}=\arg \int_{0}^{1} H\left( \pm \frac{\rho(1)}{\rho(x)}\right) d x .
$$

The upper and lower signs in formula (44) correspond to slow and fast chimeras, respectively. Remarkably, for slow chimeras Eq. (44) implies that there exists a critical value

$$
K_{c r}=\frac{\omega}{R^{+} \rho(1)},
$$

such that for $K \geq K_{c r}$ the existence of slow chimeras is not possible, while fast chimeras can be found for all positive values of $K$. Note that a similar behavior is observed for $C \neq 0$, too.

Concerning the curve at which stable and unstable chimeras disappear, one can find it as a critical line for Eq. (42). For this, one needs to consider Eq. (42) jointly with the degeneracy condition $-\tau K P^{\prime}\left(x_{0}\right)=Q^{\prime}\left(x_{0}\right)$ and exclude $x_{0}$ from the obtained system. However, in this case, the results cannot be expressed in an explicit form similar to Eq. (44).

For $C=0$, one obtains also a simpler description of the stability region for the completely incoherent state. In particular, inequalities (34) and (35) now read

$$
\frac{4 \pi m+\pi}{2 \omega-K}<\tau<\frac{4 \pi m+3 \pi}{2 \omega+K}, \quad 0<K<\frac{\omega}{2 m+1} .
$$

They define a sequence of trianglelike domains, with $m=0,1,2, \ldots$ [see Fig. 8(a)]. Taking into account the equivalence of the continuous formulation method from $[53,55]$ and the method used in the present paper, it is not surprising that expression (45) provides the boundaries of the stability region for the completely incoherent state, which coincides with the analogous Eq. (4) in [33].

\section{Conclusion}

We have demonstrated that the chimera states as well as related coherent vs incoherent solutions in systems with nonhomogeneous coupling can be effectively analyzed with the approach recently proposed by Pikovsky and Rosenblum [44]. Applying this method to a system of globally coupled oscillators with spatially modulated delayed feedback, we obtained an approximate dynamical description of all typical synchronization phenomena occurring there. In particular, we found that the standing-wave solutions of the approximating equation correspond to the synchronized and partially synchronized (chimera states) solutions of the initial system. We have clarified the link between the chimera states from one side and the coherent and incoherent solutions from the other side, considering more accurately the stability of such solutions and bifurcation transitions between them. Finally, we obtained a series of analytical and numerical results revealing possibilities of the spatially modulated delayed feedback to generate the chimera states and control their form. In particular, we showed that the control is extremely effective, when the coupling strength is small compared to the natural frequencies of oscillators [that is a typical condition for phase reduction of Eq. (1)]. In this case, varying the parameters of the delayed feedback in an appropriate region of the parameter space (typically, $\tau \sim T$ and $K \sim \omega$ ), one can induce chimera states with different sizes of coherent vs incoherent domains or with different distributions of effective frequencies. For large stimulation or coupling strengths as well as for large delays, the spatially modulated delayed feedback also produces partially synchronized solutions. But in this case, the control generates the fast chimeras only, which are in a certain sense close to the phase-locked states.

In the present paper we have considered a simple mathematical model of coupled phase oscillators. But chimera states may be relevant in other physical and biological systems, too. A fundamental problem in neuroscience is feature binding [61]: How are, e.g., different features, which belong to a meaningful signal pattern, represented and coded by the brain? It has been suggested that the neurons which represent the single features join into one synchronized ensemble (of coincidently firing neurons) in order to establish a represen- 
tation of the pattern as a whole [61]. However, in the simple model we have considered here an input into an oscillatory network (i.e., the delayed feedback) may induce chimeras, where the synchronized regime and the desynchronized regime coexist and are mutually dependent. Hence, by exclusively focusing on synchronization we would have missed the superordinate organization of the whole process, the chimera. In fact, the relationship between model parameters and external input is not adequately reflected by only considering the portion of synchronized oscillators. Rather all dynamical features of the chimera states considered above (see, e.g., Fig. 9) have to be taken into account to characterize the "response" of the oscillator network to the external input (delayed feedback): which spatial part of the network gets synchronized and what is the frequency distribution? Accordingly, anatomically distributed and well-tuned mixtures of synchronization and incoherency might be an alternative and possibly more robust candidate mechanism for feature binding.

\section{ACKNOWLEDGMENTS}

This study was supported by the EU Network of Excellence in Biosimulation (Contract No. LSHB-CT-20004005137). We also acknowledge valuable discussions with Matthias Wolfrum and Arkady Pikovsky.
[1] Y. Kuramoto, Chemical Oscillations, Waves, and Turbulence (Springer, Berlin, 1984).

[2] S. H. Strogatz, Physica D 143, 1 (2000).

[3] A. Winfree, The Geometry of Biological Time (Springer, Berlin, 2001).

[4] A. Pikovsky, M. Rosenblum, and J. Kurths, Synchronization: A Universal Concept in Nonlinear Sciences (Cambridge University Press, Cambridge, England, 2001).

[5] E. Mosekilde, Y. Maistrenko, and D. Postnov, Chaotic Synchronization: Application to Living Systems (World Scientific, Singapore, 2002).

[6] P. A. Tass, Phase Resetting in Medicine and Biology (Springer, Berlin, 1999).

[7] S. Boccaletti, J. Kurths, G. Osipov, D. L. Valladares, and C. S. Zhou, Phys. Rep. 366, 1 (2002).

[8] G. Kozyreff, A. G. Vladimirov, and P. Mandel, Phys. Rev. Lett. 85, 3809 (2000).

[9] P. Ashwin, J. Buescu, and I. Stewart, Nonlinearity 9, 703 (1996).

[10] V. N. Belykh, I. V. Belykh, and M. Hasler, Phys. Rev. E 62, 6332 (2000)

[11] W. Wang, I. Z. Kiss, and J. L. Hudson, Chaos 10, 248 (2000).

[12] Y. Maistrenko, O. Popovych, O. Burylko, and P. A. Tass, Phys. Rev. Lett. 93, 084102 (2004).

[13] D. Pazó, Phys. Rev. E 72, 046211 (2005).

[14] Y. Kuramoto and D. Battogtokh, Nonlinear Phenom. Complex Syst. (Dordrecht, Neth.) 5, 380 (2002).

[15] Y. Kuramoto, in Nonlinear Dynamics and Chaos: Where Do We Go from Here? edited by S. J. Hogan, A. R. Champneys, B. Krauskopf, M. di Bernardo, R. E. Wilson, H. M. Osinga, and M. E. Homer (Institute of Physics, Bristol, 2003), p. 209.

[16] S. I. Shima and Y. Kuramoto, Phys. Rev. E 69, 036213 (2004).

[17] D. M. Abrams and S. H. Strogatz, Phys. Rev. Lett. 93, 174102 (2004).

[18] D. M. Abrams and S. H. Strogatz, Int. J. Bifurcation Chaos 16, 21 (2006).

[19] Y. Kawamura, Phys. Rev. E 75, 056204 (2007).

[20] G. C. Sethia, A. Sen, and F. M. Atay, Phys. Rev. Lett. 100, 144102 (2008).

[21] C. R. Laing, Chaos 19, 013113 (2009).

[22] C. R. Laing, Physica D 238, 1569 (2009).

[23] A. E. Motter, Nat. Phys. 6, 164 (2010).
[24] E. A. Martens, C. R. Laing, and S. H. Strogatz, Phys. Rev. Lett. 104, 044101 (2010).

[25] O. E. Omel'chenko, M. Wolfrum, and Y. L. Maistrenko, Phys. Rev. E 81, 065201(R) (2010).

[26] G. Bordyugov, A. Pikovsky, and M. Rosenblum, Phys. Rev. E 82, 035205 (2010).

[27] O. E. Omel'chenko, Y. L. Maistrenko, and P. A. Tass, Phys. Rev. Lett. 100, 044105 (2008).

[28] D. M. Abrams, R. Mirollo, S. H. Strogatz, and D. A. Wiley, Phys. Rev. Lett. 101, 084103 (2008).

[29] D. M. Abrams, R. Mirollo, S. H. Strogatz, and D. A. Wiley, Phys. Rev. Lett. 101, 129902 (2008).

[30] J. H. Sheeba, V. K. Chandrasekar, and M. Lakshmanan, Phys. Rev. E 79, 055203 (2009).

[31] C. R. Laing, Phys. Rev. E 81, 066221 (2010).

[32] E. A. Martens, Phys. Rev. E 82, 016216 (2010).

[33] M. K. Stephen Yeung and S. H. Strogatz, Phys. Rev. Lett. 82, 648 (1999)

[34] M. G. Rosenblum and A. S. Pikovsky, Phys. Rev. Lett. 92, 114102 (2004).

[35] O. V. Popovych, C. Hauptmann, and P. A. Tass, Phys. Rev. Lett. 94, 164102 (2005).

[36] N. B. Janson, A. G. Balanov, and E. Schöll, Phys. Rev. Lett. 93, 010601 (2004).

[37] B. Hauschildt, N. B. Janson, A. Balanov, and E. Schöll, Phys. Rev. E 74, 051906 (2006).

[38] A. G. Balanov, V. Beato, N. B. Janson, H. Engel, and E. Schöll, Phys. Rev. E 74, 016214 (2006).

[39] C. Hauptmann, O. Omel'chenko, O. V. Popovych, Y. Maistrenko, and P. A. Tass, Phys. Rev. E 76, 066209 (2007).

[40] O. E. Omel'chenko, C. Hauptmann, Y. L. Maistrenko, and P. A. Tass, Physica D 237, 365 (2008).

[41] N. Yousif and X. Liu, Expert Rev. Med. Devices 4, 623 (2007).

[42] B. Fiedler, S. Yanchuk, V. Flunkert, P. Hövel, H.-J. Wünsche, and E. Schöll, Phys. Rev. E 77, 066207 (2008).

[43] S. Yanchuk and P. Perlikowski, Phys. Rev. E 79, 046221 (2009).

[44] A. Pikovsky and M. Rosenblum, Phys. Rev. Lett. 101, 264103 (2008).

[45] P. A. Tass and H. Haken, Z. Phys. B: Condens. Matter 100, 303 (1996) 
[46] S. Watanabe and S. H. Strogatz, Phys. Rev. Lett. 70, 2391 (1993).

[47] S. Watanabe and S. H. Strogatz, Physica D 74, 197 (1994).

[48] E. Ott and T. M. Antonsen, Chaos 18, 037113 (2008).

[49] E. Ott and T. M. Antonsen, Chaos 19, 023117 (2009).

[50] J. K. Hale and S. M. V. Lunel, Introduction to Functional Differential Equations (Springer, Berlin, 1993).

[51] R. E. Mirollo and S. H. Strogatz, Physica D 205, 249 (1995).

[52] S. Yanchuk and M. Wolfrum, Phys. Rev. E 77, 026212 (2008).

[53] S. H. Strogatz and R. E. Mirollo, J. Stat. Phys. 63, 613 (1991).

[54] R. Mirollo and S. H. Strogatz, J. Nonlinear Sci. 17, 309 (2007).

[55] S. H. Strogatz, R. E. Mirollo, and P. C. Matthews, Phys. Rev. Lett. 68, 2730 (1992).

[56] J. Xu and K. W. Chung, Physica D 180, 17 (2003).

[57] A. G. Balanov, N. B. Janson, and E. Schöll, Phys. Rev. E 71, 016222 (2005).

[58] Solving the discretized spectral problem (28), we obtain in this case the pseudocontinuous spectrum which is separated from the imaginary axis and lies completely in the LHP. However, we presume that for larger delays the behavior of the spectrum may be different. In particular, the pseudocontinuous spectrum may cross the imaginary axis, resulting in a Turing-like bifurcation scenario for chimera states. We plan to address this issue in a future study.

[59] We recall that in our settings the chimera state is described as a standing-wave solution to system (12) and (13); therefore, we use here the term "torus bifurcation" to distinguish this bifurcation of a periodic orbit from the classical Hopf bifurcation of a fixed point. The latter also appears in our analysis, but for the completely incoherent state.

[60] Note that the result of the dynamical simulation of the finitesize system (5) is not sensitive to the presence of a small amount of noise, provided we observe phase-locked states and chimera states only. However, the presence of noise is crucial if we want to resolve the stability properties of the completely incoherent state.

[61] W. Singer and C. M. Gray, Annu. Rev. Neurosci. 18, 555 (1995). 